\title{
Comportamiento mecánico de concreto fabricado con agregados reciclados
}

\author{
I.E. Martínez-Soto y C.J. Mendoza-Escobedo \\ Instituto de Ingeniería, UNAM \\ E-mails: irismartinezs@yahoo.com y cjm@ pumas.iingen.unam.mx
}

(Recibido: mayo de 2005; aceptado: noviembre de 2005)

\section{Resumen}

El desperdicio producido por las plantas premezcladoras de concreto representa un problema de residuos sólidos que necesita solución. Este concreto puede ser utilizado para fabricar agregados. En este trabajo, se presenta el desempeño de concretos fabricados con agregados reciclados obtenidos a partir de cilindros de concreto premezclado y diferentes consumos de cemento. Los resultados experimentales mostraron que el comportamiento del concreto con agregados reciclados es similar al del concreto con agregados naturales, lo que sugiere que puede ser utilizado como un concreto clase dos, de acuerdo con el Reglamento de Construcción del Distrito Federal (RCDF).

Descriptores: Agregado reciclado, concreto reciclado, propiedades mecánicas del concreto.

\begin{abstract}
The sol id wastes pro duced by the ready mixed con crete in dus try rep resent a se ri ous prob lem that need an im medi ately solu tion. This con cretemight be used to fab ri cate recy cled con creteag gregate (RCA). In this pa per themechan i cal prop er ties of con cretefab ri cated with RCA and dif fer ent ce ment con tents are presented. The RCA werefab ri cated using old lab o ra tory speci mens madeof ready mixed con crete. Theresults showed theprop er ties of therecy cled con cretearesim il ar to that of nat u ral con crete. A c cord ingly, the recy cled con cretecan likely be used as typetwo concrete in agree ment with $\mathrm{M}$ ex ico C ity Build ing Code (RCDF).
\end{abstract}

Keywords: Recy cled con creteag gregate, recy cled con crete, mechan i cal prop er ties of thecon crete.

\section{Introducción}

Las plantas premezcladoras de concreto generan un porcentaje importante de desperdicio (estimado en un 10\%) del concreto producido. Esto representa una pérdida de las materias primas que componen el concreto, principalmente del agregado natural, ya que este insumo es un recurso natural no renovable. Además, este desperdicio también implica la necesidad de un sitio para su disposición final, lo que a su vez, representa un problema debido a la escasez de áreas disponibles para ello. Por lo tanto, resulta imperativo dar una solución al problema. En este trabajo se propone el reciclaje del concreto premezclado para fabricar agregados gruesos como una posible solución. Para mostrar la factibilidad de la propuesta, en esta investigación se evaluaron las propiedades mecánicas de concretos elaborados con agregados gruesos producto del reciclaje de especímenes de concreto premezclado, y se compararon, ante igualdad de condiciones de fabricación, con las propiedades de concretos naturales (hechos con agregados naturales). Las propiedades mecánicas comparadas fueron el módulo de elasticidad y las resistencias a la compresión, tensión y flexión, todas obtenidas mediante pruebas de laboratorio. Los resultados experimen- 
Comportamiento mecánico de concreto fabricado con agregados reciclados

tales obtenidos mostraron que los agregados, producto del reciclaje de concreto premezclado, producen concretos reciclados que pueden utilizarse como concretos clase dos, de acuerdo con el Reglamento de Construcción del Distrito Federal (RCDF, 2003). Estos resultados muestran la factibilidad del reciclaje de concreto como una solución al problema, reutilizando el concreto premezclado y el obtenido de la demolición de estructuras existentes.

\section{Agregados}

En este trabajo, los agregados gruesos naturales utilizados fueron calizas, y a éstos se les llama como tal (agregados naturales). Por otra parte, a los agregados gruesos producto de la trituración de concreto premezclado, se les llama agregados reciclados. Estos agregados son también de caliza, ya que se tuvo especial cuidado de que el concreto premezclado utilizado para fabricarlos, estuviera compuesto solamente de caliza natural. Respecto del agregado fino, sólo se utilizó arena andesítica natural.
Para los dos tipos de agregados gruesos, naturales y reciclados, se consideró un tamaño máximo de agregado (TMA) de $19 \mathrm{~mm}$ (3/4"). De acuerdo con este TMA, se utilizó un intervalo granulométrico correspondiente a un material bien graduado, según la norma ASTM C 33.

\section{Fabricación}

La granulometría original de los agregados naturales estaba fuera de los límites establecidos en la norma ASTM C 33 para el TMA considerado, por lo que fue necesario triturar el material para recomponer sus tamaños y cumplir así con la norma. Esta recomposición de tamaños se hizo considerando una proporción del 35\% de partículas que pasan la malla de $9.5 \mathrm{~mm}\left(3 / 8^{\prime \prime}\right)$ y $65 \%$ que pasan la malla de $19 \mathrm{~mm}$ (3/4"). Esta proporción permitió cumplir con lo establecido en la norma ASTM C 33.

El proceso de recomposición granulométrica aplicado a los agregados naturales se muestra en la figura 1.

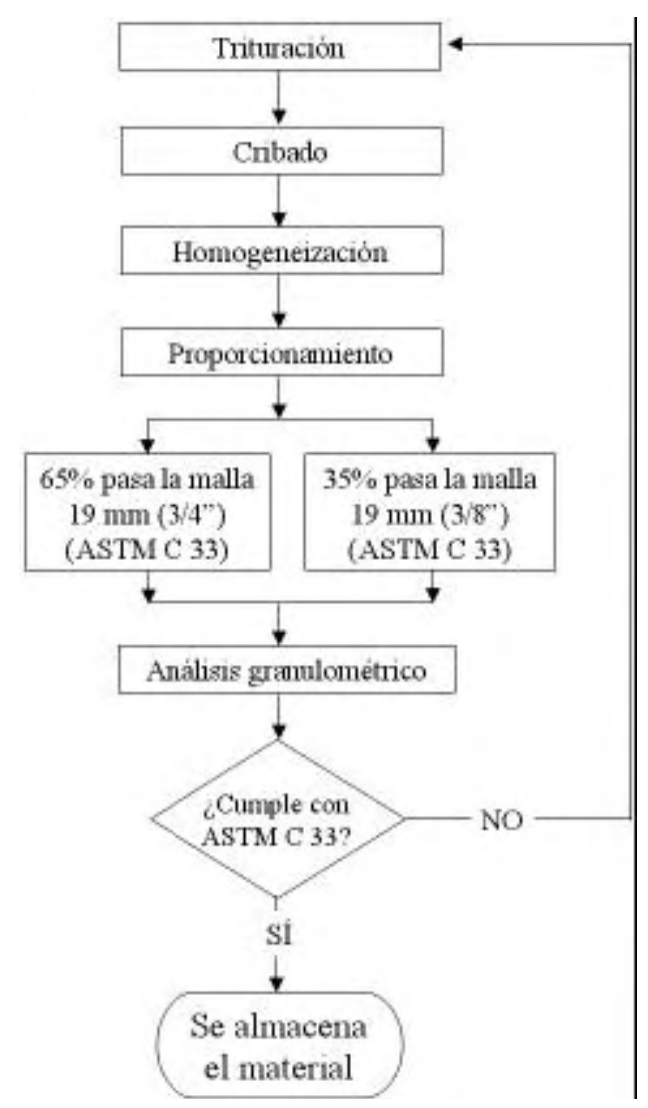

Figura 1. Proceso de fabricación de agregados 
El equipo de trituración utilizado fue la trituradora eléctrica de muelas del laboratorio de materiales del Instituto de Ingeniería, UNAM (IIUNAM), la cual acepta un TMA de $75 \mathrm{~mm}$ (3"). Para el cribado del material, se utilizó la criba eléctrica del laboratorio de mecánica de rocas del IIUNAM. Este equipo cuenta con las mallas de $19 \mathrm{~mm}\left(3 / 4^{\prime \prime}\right)$, la de 9.5 $\mathrm{mm}\left(3 / 8^{\prime \prime}\right)$, y la de $4.76 \mathrm{~mm}$ (\#4). La criba entrega por separado cuatro tipos de materiales: retenido $19 \mathrm{~mm}$ (R19), pasa la $19 \mathrm{~mm}$ (P19), pasa la $9.5 \mathrm{~mm}$ (P9.5) y el que pasa la malla de $4.76 \mathrm{~mm}$ (P4.76).

Los tamaños utilizados para la recomposición granulométrica fueron el P19 y el P9.5.

Respecto de los agregados reciclados, éstos se fabricaron de acuerdo al mismo procedimiento de trituración y cribado utilizado en los agregados naturales (Figura 1), con la diferencia de que la materia prima fue concreto premezclado. En este caso se realizó una trituración primaria manual y posteriormente se siguió con el proceso de fabricación mostrado en la figura 1.

\section{Propiedades físicas}

Las propiedades físicas estudiadas para los agregados gruesos fueron la granulometría, co eficiente de forma, humedad, absorción, densidad relativa y peso volumétrico compactado. Los resultados se presentan en conjunto para los agregados naturales y reciclados con fines de comparación.

Las propiedades físicas estudiadas para el agregado fino fueron la granulometría, módulo de finura, humedad, absorción, densidad relativa y peso volumétrico compactado.

\section{Granulometría}

La granulometría de los agregados gruesos, naturales y reciclados, se controló de acuerdo a la norma ASTM C 33 para tener las mismas condiciones y así tener una base de comparación. En la figura 2 se muestran las curvas granulométricas de los dos tipos de agregados gruesos.

En el caso del agregado fino, no se hizo una corrección granulométrica, ya que se consideró que su granulometría era aceptable para ser utilizada en las mezclas de concreto. La figura 3 muestra la curva granulométrica de la arena.

El módulo de finura de la arena se obtuvo de acuerdo con la norma ASTM C 125. El valor fue de 3.15 , lo que indica que la arena es gruesa.

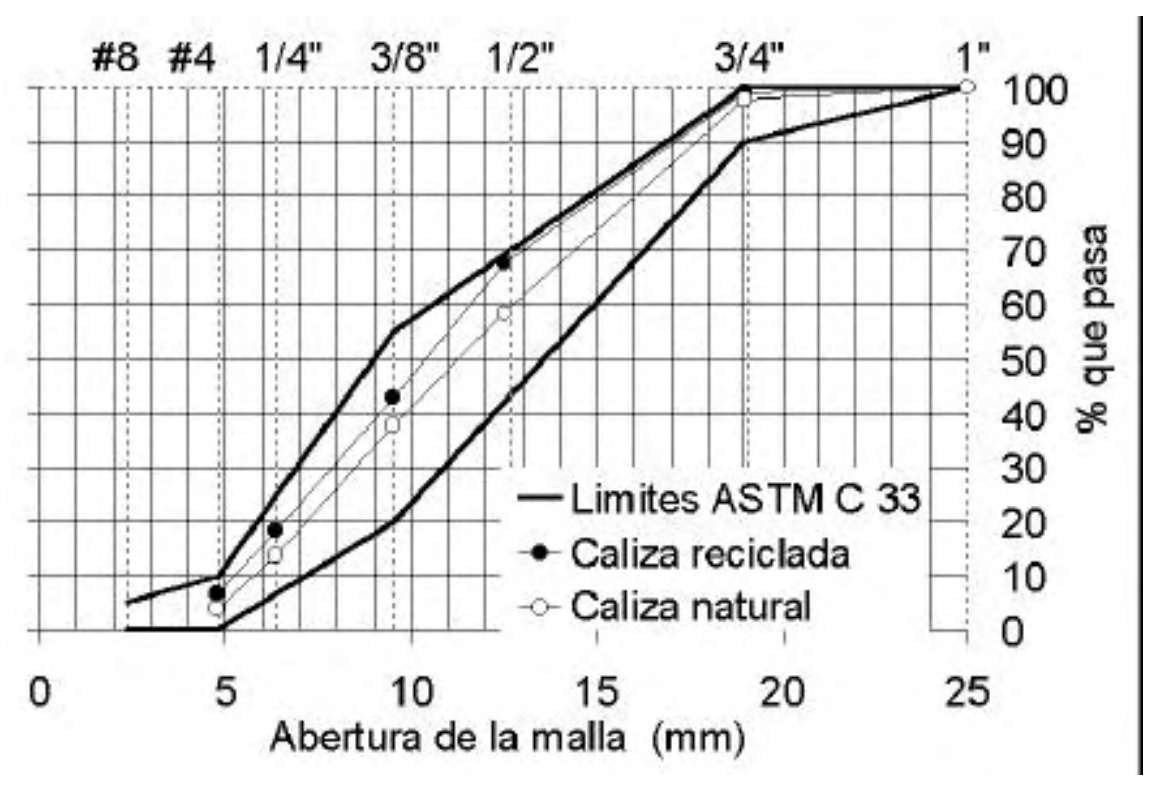

Figura 2. Granulometría de agregados gruesos 


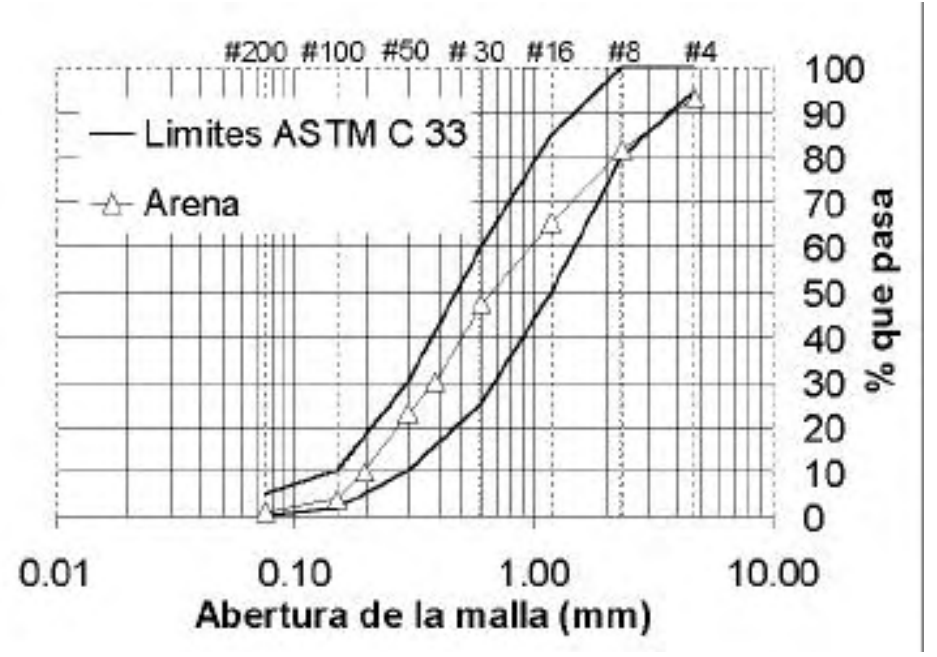

Figura 3. Granulometría de la arena

\section{Coeficiente de forma}

El cálculo del coeficiente de forma se hizo de acuerdo con la norma francesa NFP 18-301. La tabla 1 presenta los resultados obtenidos. En esta tabla se observa que el coeficiente de forma de los agregados gruesos naturales y reciclados es similar, lo cual era de esperarse debido a que ambos materiales son producto de trituración. La forma de las partículas de ambos agregados cumple los límites establecidos por el RCDF.

Tabla 1. Coeficientes de forma de los agregados gruesos (adimensional)

\begin{tabular}{rc}
\hline Agregado & Coeficiente de forma \\
\hline Natural & 0.20 \\
Reciclado & 0.24 \\
\hline
\end{tabular}

\section{Humedad}

La humedad que todos los agregados (naturales, reciclados y arena) tenían en el momento de los experimentos se midió siguiendo el procedimiento establecido en la norma ASTM C 566. Los valores obtenidos se presentan en la tabla 2 . Se observa claramente que los agregados gruesos reciclados tenían una humedad mayor que la de los naturales. Estos valores de humedad se utilizaron para realizar los proporcionamientos de las mezclas de concreto, ya que se previnieron variaciones en las mismas.
Tabla 2. H umedades de los agregados

\begin{tabular}{cc}
\hline Agregado & Humedad (\%) \\
\hline Natural & 0.04 \\
Reciclado & 3.33 \\
Arena & 6.93 \\
\hline
\end{tabular}

\section{Absorción}

Esta propiedad se midió de acuerdo con la norma ASTM C 127 y 128, para agregados gruesos y finos, respectivamente. Los resultados se muestran en la tabla 3. Es evidente que los agregados gruesos reciclados son notablemente más absorbentes que los naturales. Sin embargo, ambos están dentro de los rangos normales, al igual que la arena.

Tabla 3. Absorción de los agregados

\begin{tabular}{cc}
\hline Agregado & Absorción (\%) \\
\hline Natural & 0.42 \\
Reciclado & 2.62 \\
Arena & 6.03 \\
\hline
\end{tabular}

\section{Densidad relativa}

La densidad relativa de los agregados se midió de acuerdo con las normas ASTM C 127 y 128, para agregados gruesos y finos, respectivamente. En la tabla 4 se presentan los resultados. Se aprecia que 
la densidad de los agregados reciclados fue menor que la de los naturales, lo cual podía esperarse, ya que el contenido de pasta de cemento en el material reciclado disminuye la densidad global del agregado.

Tabla 4. D ensidad relativa (adimensional)

\begin{tabular}{cc}
\hline Agregado & Densidad \\
\hline Natural & 2.71 \\
Reciclado & 2.36 \\
Arena & 2.31 \\
\hline
\end{tabular}

El intervalo normal de densidad relativa para la mayoría de los agregados naturales es de 2.40 2.90 (Kosmatka et al, 1992), por lo que el agregado reciclado tiene una densidad relativa ligeramente baja respecto a la de un agregado natural. Sin embargo, el valor medido en el laboratorio (2.36) es muy bueno para ser un agregado reciclado ya que está muy próximo al rango de variación de los agregados naturales.

\section{Peso volumétrico compactado}

Esta propiedad se midió de acuerdo con la norma ASTM C 29. De acuerdo con Kosmatka (1992), el peso volumétrico compactado de un agregado usado para concreto de peso normal, varía de 1200 a $1760 \mathrm{~kg} / \mathrm{m}^{3}$. Los valores obtenidos en el laboratorio están dentro de este intervalo (Tabla 5).

Tabla 5. Peso volumétrico compactado

\begin{tabular}{rc}
\hline Agregado & Peso Vol. $\left(\mathrm{kg} / \mathrm{m}^{3}\right)$ \\
\hline Natural & 1563 \\
Reciclado & 1309 \\
Arena & 1584 \\
\hline
\end{tabular}

\section{Elaboración de concretos}

De aquí en adelante, a los concretos fabricados con los agregados naturales se les llama "concretos naturales", y a los fabricados con agregados reciclados se les llama "concretos reciclados". En ambos tipos de concreto se utilizó arena natural de andesita con las propiedades físicas descritas anteriormente.

\section{Proporcionamientos}

Para la elaboración de las mezclas de concreto se utilizó un procedimiento modificado a partir del método ACl 211 (Martínez-Soto, 2005).

El procedimiento seguido fue el siguiente: primero se fijaron los consumos de cemento a utilizar en cada mezcla, es decir, 200, 300 y $400 \mathrm{~kg} / \mathrm{m}^{3}$ para los dos tipos de agregados, naturales y reciclados. A partir del TMA considerado y el revenimiento fijado para todas las mezclas en $10 \mathrm{~cm}$, se encontró un consumo de agua establecido por el método $\mathrm{ACl}$. Después se definieron los volúmenes absolutos en porcentaje para agregados finos y gruesos que se consideraron congruentes para cada consumo de cemento, ya que el volumen de agregado grueso que establece el método $\mathrm{ACl}$, era el mismo para todas las mezclas, pues depende del módulo de finura de la arena. En la figura 4 se muestra el procedimiento de dosificación de las mezclas.

Las mezclas resultado de estas proporciones se nombraron de la siguiente manera: las elaboradas con agregado de caliza natural se etiquetaron como CN200, CN300 y CN400, y las elaboradas con agregado de caliza reciclada como CR200, CR300 y CR400 para cada consumo de cemento de 200,300 y $400 \mathrm{~kg} / \mathrm{m}^{3}$, respectivamente.

En la tabla 6 se presentan las cantidades correspondientes al agua de mezclado utilizada y a la cantidad de cemento ajustadas al peso volumétrico obtenido en el laboratorio, ya que el rendimiento de la mezcla en muchos casos fue ligeramente mayor que uno. Cuando se observa la tabla 6 , es posible notar que los porcentajes de agregado que se muestran en la figura 4, no corresponde directamente a los pesos de agregados mostrados en la tabla, principalmente en los naturales. Esto se debe a que los agregados gruesos naturales tienen un peso específico mucho mayor que la arena utilizada, mientras que los agregados reciclados tanto finos como gruesos, tienen pesos específicos muy semejantes, además las cantidades presentadas están afectadas por la humedad que el material contenía en el momento que se hicieron los proporcionamientos. 
Comportamiento mecánico de concreto fabricado con agregados reciclados

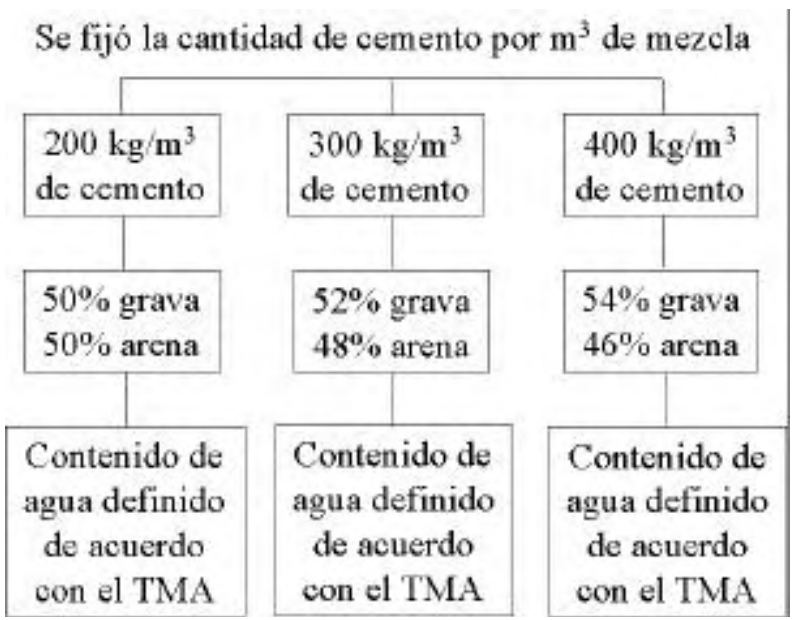

Figura 4. Criterio de dosificación de las mezclas de concreto

Tabla 6. Proporcionamientos de las mezclas de concreto

\begin{tabular}{ccccc}
\hline Mezcla & Agua $\left(\mathrm{kg} / \mathrm{m}^{3}\right)$ & Cemento $\left(\mathrm{kg} / \mathrm{m}^{3}\right)$ & Grava $\left(\mathrm{kg} / \mathrm{m}^{3}\right)$ & Arena $\left(\mathrm{kg} / \mathrm{m}^{3}\right)$ \\
\hline CN200 & 200.37 & 198.38 & 961.75 & 886.18 \\
CN300 & 211.56 & 293.80 & 943.19 & 802.23 \\
CN400 & 216.49 & 387.33 & 922.94 & 724.43 \\
CR200 & 247.02 & 189.79 & 838.45 & 848.78 \\
CR300 & 240.57 & 281.75 & 822.67 & 770.71 \\
CR400 & 252.08 & 371.16 & 803.03 & 695.93 \\
\hline
\end{tabular}

\section{Ensayes de laboratorio}

Los ensayes de laboratorio practicados a los concretos elaborados, fueron en estado fresco: el revenimiento, el peso volumétrico fresco y el contenido de aire, y en estado endurecido: las resistencias a compresión, a tensión y a flexión, así como el módulo de elasticidad, según los procedimientos establecidos en las normas ASTM correspondientes.

Para el estudio de estas propiedades se fabricaron cilindros y vigas de concreto estándar de $0.15 \times 0.30 \mathrm{~m}$ y $0.15 \times 0.15 \times 0.60 \mathrm{~m}$, respectivamente.

Las propiedades de resistencia a la compresión se estudiaron a las edades de 7, 14 y 28 días, mientras que el resto de los ensayes se estudiaron para 28 días solamente.

\section{Resultados y discusión}

Propiedades en estado fresco

\section{Revenimiento}

Para obtener el revenimiento de las mezclas de concreto se utilizó el procedimiento establecido en la norma ASTM C 143. En la tabla 7 se presentan los revenimientos obtenidos. Como se puede apreciar la mayoría de los revenimientos se encuentran dentro de los límites de tolerancia $( \pm$ $2.5 \mathrm{~cm}$ ) que están establecidos en la fracción 6.1.2 de la norma ASTM C 94. Sólo la mezcla CR300 estuvo fuera de los límites.

Los resultados de otras investigaciones similares muestran que la trabajabilidad de las mezclas recicladas es menor que la de las mezclas naturales (e.g., Sagoe, 2001). Los resultados 
I.E. Martínez-Soto y C.J. Mendoza-Escobedo

obtenidos en este trabajo concuerdan con esta tendencia generalizada, según se observa en la figura 5 donde se presentan los valores de revenimiento para los concretos reciclados y naturales.

Tabla 7. Revenimientos obtenidos

\begin{tabular}{cc}
\hline Mezcla & Revenimiento $(\mathrm{cm})$ \\
\hline CN200 & 8.87 \\
CR200 & 8.10 \\
CN300 & 10.00 \\
CR300 & 6.93 \\
CN400 & 9.17 \\
CR400 & 8.65 \\
\hline
\end{tabular}

\section{Peso volumétrico fresco}

Los pesos volumétricos frescos obtenidos de acuerdo con el procedimiento establecido en la norma ASTM C 138, se presentan en la tabla 8. En ésta se observa que los pesos volumétricos de los concretos reciclados fueron bajos en relación con los que alcanzaron los naturales. Lo cual era de esperarse. Otras investigaciones realizadas (Sagoe, 2001) con agregado reciclado de mayor peso específico como el basalto, han reportado mezclas recicladas de pesos volumétricos mayores a los aquí obtenidos. Esto es congruente con los resultados presentados en este trabajo donde se utilizó agregado reciclado de caliza.
Tabla 8. Pesos volumétricos frescos

\begin{tabular}{cc}
\hline Mezcla & Peso volumétrico fresco $\left(\mathrm{kg} / \mathrm{m}^{3}\right)$ \\
\hline CN200 & 2246.68 \\
CR200 & 2124.05 \\
CN300 & 2250.78 \\
CR300 & 2115.70 \\
CN400 & 2251.20 \\
CR400 & 2122.21 \\
\hline
\end{tabular}

Por otra parte, según lo establecido en el RCDF, este concreto reciclado se clasifica como concretos clase dos, ya que tiene pesos volumétricos frescos de 1,900 a $2,200 \mathrm{~kg} / \mathrm{m}^{3}$, mientras que los naturales están clasificados como concretos clase uno por tener pesos volumétricos frescos mayores a $2,200 \mathrm{~kg} / \mathrm{m}^{3}$.

\section{Contenido de aire}

El contenido de aire de las mezclas de concreto se obtuvo de acuerdo con el procedimiento que se establece en la norma ASTM C 231. En la tabla 9 se presentan los contenidos de aire obtenidos de las seis mezclas elaboradas.

En esta tabla se observa que todas las mezclas tuvieron contenidos de aire normales entre, el 1.5, y $2.0 \%$, sólo la mezcla CR300 obtuvo un contenido de aire ligeramente mayor.

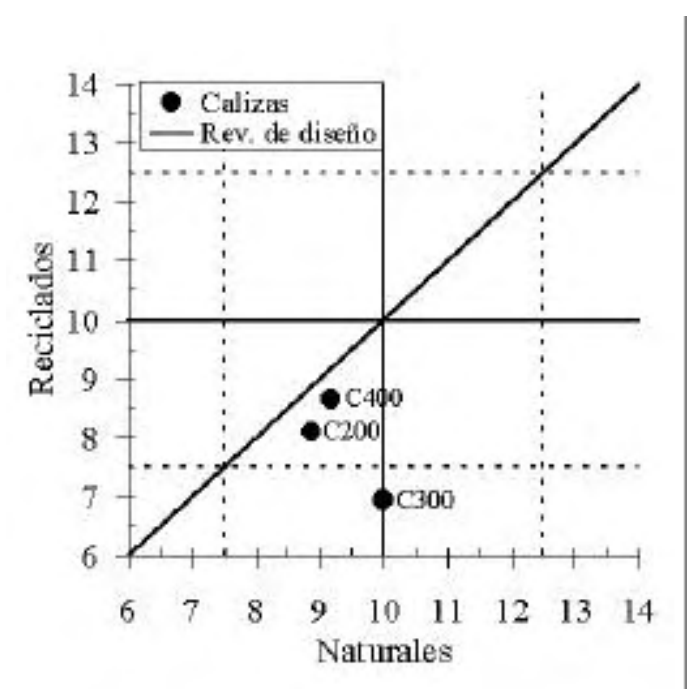

Figura 5. Posicionamiento de revenimientos reciclados-naturales 
Tabla 9. Contenidos de aire obtenidos

\begin{tabular}{lc}
\hline Mezcla & Contenido de aire (\%) \\
\hline CN200 & 2.00 \\
CR200 & 1.80 \\
CN300 & 2.00 \\
CR300 & 2.10 \\
CN400 & 2.00 \\
CR400 & 2.00 \\
\hline
\end{tabular}

\section{Propiedades en estado endurecido}

\section{Resistencia a la compresión}

La resistencia a la compresión de las mezclas de concreto se obtuvo siguiendo el procedimiento establecido en la norma ASTM C 39. Las resistencias a la compresión obtenidas se presentan en la tabla 10.

Tabla 10. Resistencias a la compresión de los concretos

\begin{tabular}{ccccc}
\hline Mezcla & $\begin{array}{c}\text { Relación } \\
\text { a/c }\end{array}$ & \multicolumn{3}{c}{$\begin{array}{c}\text { Resistencia a la compresión } \\
\left(\mathrm{kg} / \mathrm{cm}^{2}\right)\end{array}$} \\
\hline & & $7 \mathrm{~d}$ & $14 \mathrm{~d}$ & $28 \mathrm{~d}$ \\
\cline { 3 - 5 } CN200 & 1.01 & 116.70 & 145.08 & 188.20 \\
CR200 & 1.30 & 103.52 & 140.48 & 185.53 \\
CN300 & 0.72 & 218.20 & 252.25 & 306.86 \\
CR300 & 0.85 & 209.97 & 266.99 & 300.73 \\
CN400 & 0.56 & 286.85 & 330.78 & 385.61 \\
CR400 & 0.68 & 256.43 & 281.63 & 360.36 \\
\hline
\end{tabular}

Si se representan las relaciones agua-cemento (a/c) y las resistencias a la compresión (fc) a 28 días en una gráfica, se observa que los concretos reciclados tienen mayores resistencias respecto de los naturales para una misma relación a/c. Hay que tomar en cuenta que los concretos reciclados requieren de mayores consumos de agua que los naturales para alcanzar revenimientos similares (Figura 6). En general, en la literatura técnica relacionada se han publicado valores de resistencia a la compresión simple, menores a los aquí obtenidos. Sin embargo, las referencias disponibles hasta el momento (Sagoe, 2001; Katz, 2003) no presentan los valores de sus concretos naturales homólogos, por lo que no es posible saber si la situación obtenida en esta investigación ha sido obtenida por otros autores.

Ahora, si se relaciona el consumo de cemento con la resistencia a la compresión obtenida, se puede observar que cuando se tiene un consumo de hasta $300 \mathrm{~kg} / \mathrm{m}^{3}$ de cemento, la eficiencia en los concretos reciclados es mayor que en los naturales, mientras que para consumos mayores a 300 $\mathrm{kg} / \mathrm{m}^{3}$ la eficiencia es mayor para los concretos naturales como se observa en la figura 7 , aunque las diferencias son pequeñas.

\section{Resistencia a la tensión}

Para un concreto de peso normal, la resistencia a la tensión se estima en 1.20 a 1.50 veces la raíz cuadrada de la resistencia a la compresión, para concretos clase dos y clase uno, respectivamente (RCDF). Esta propiedad se obtuvo siguiendo el

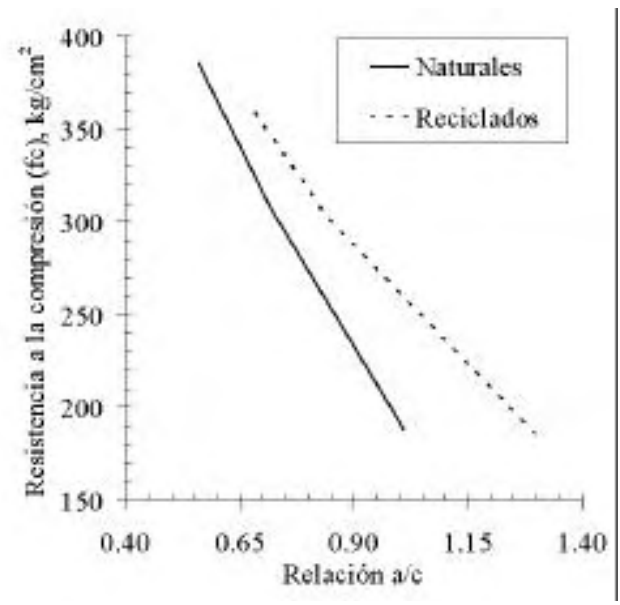

Figura 6. Curvas fc-relación a/c 
I.E. Martínez-Soto y C.J. Mendoza-Escobedo

procedimiento establecido en la norma ASTM C 496. Las resistencias a la tensión que alcanzaron las mezclas de concreto se presentan en la tabla 11. Como se puede observar en esta tabla, los concretos reciclados alcanzaron valores de resistencia a la tensión, ligeramente menores que los concretos naturales: $96 \%$ para el CR200, 92\% para el CR300 y $84 \%$ para el CR400. Abdol et al., (2001) obtuvo la misma tendencia en sus investigaciones con concretos reciclados de caliza, lo cual respalda los resultados obtenidos en esta investigación.

Al representar en una gráfica los valores obtenidos y los establecidos en el reglamento como tendencias centrales, se puede observar que los concretos reciclados pueden considerarse dentro de la clasificación para concretos clase dos, mientras que los naturales como concretos clase uno (Figura 8).

\section{Resistencia a la flexión}

La resistencia a la flexión o módulo de ruptura para un concreto de peso normal se considera como 2 y 1.4 veces la raíz cuadrada de la resistencia a la compresión para concretos clase uno y clase dos, respectivamente. Para obtener los valores de resistencia a la flexión que alcanzaron los concretos, se siguió el procedimiento establecido en la norma ASTM C 78.

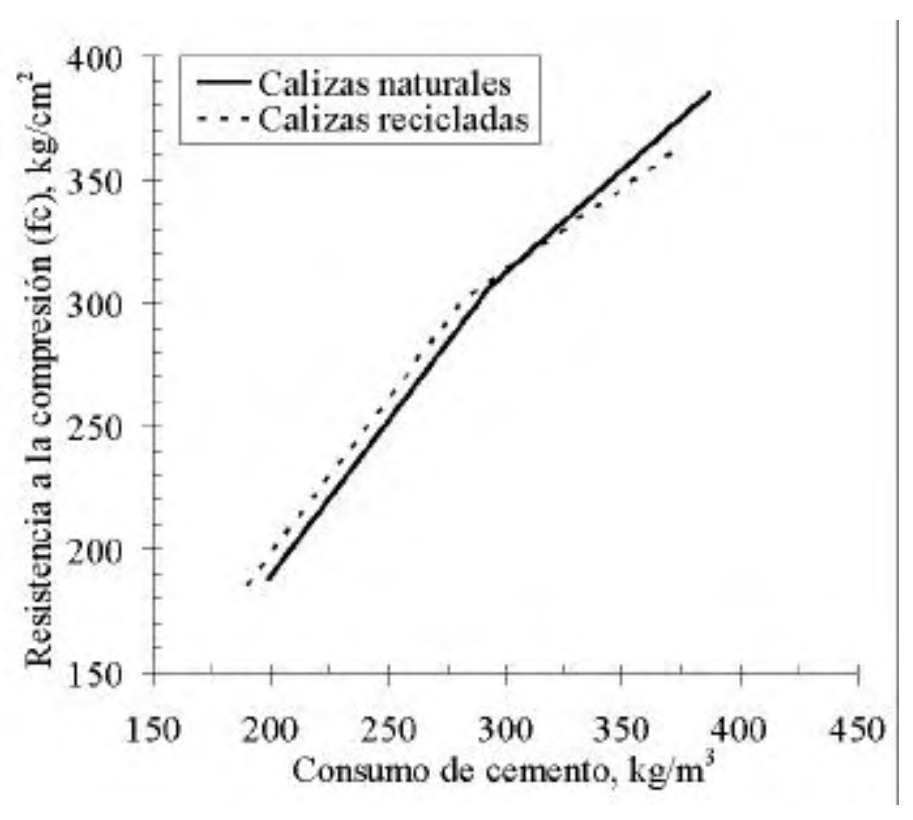

Figura 7. E ficiencia en el consumo de cemento

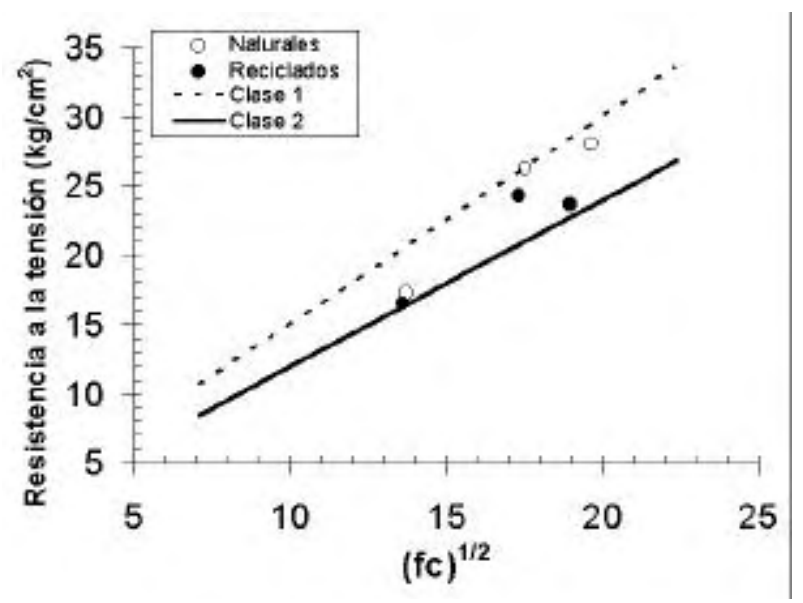

Figura 8. Resistencias a la tensión alcanzadas 
Comportamiento mecánico de concreto fabricado con agregados reciclados

Tabla 11. Resistencias a la tensión de los concretos

\begin{tabular}{lccc}
\hline Mezcla & $\begin{array}{c}\text { Resistencia } \\
\text { a la tensión } \\
(\mathrm{ft}) \\
\left(\mathrm{kg} / \mathrm{cm}^{2}\right)\end{array}$ & $\begin{array}{c}\text { Concreto } \\
\text { clase 1 } \\
1.5 \times(\mathrm{fc}))^{2} / 2\end{array}$ & $\begin{array}{c}\text { Concreto } \\
\text { clase 2 } \\
1.2 \times(\mathrm{fc}){ }^{2 / 2}\end{array}$ \\
\hline CN200 & 17.23 & 20.58 & 16.46 \\
CR200 & 16.55 & 20.43 & 16.34 \\
CN300 & 26.30 & 26.28 & 21.02 \\
CR300 & 24.23 & 26.01 & 20.81 \\
CN400 & 28.04 & 29.46 & 23.57 \\
CR400 & 23.63 & 28.47 & 22.78 \\
\hline
\end{tabular}

En la tabla 12 se presentan las resistencias a la flexión de los concretos estudiados.

Tabla 12. Resistencias a la flexión de los concretos

\begin{tabular}{lccc}
\hline Mezcla & $\begin{array}{c}\text { Resistencia } \\
\text { a la flexión } \\
(\mathrm{MR}) \\
\left(\mathrm{kg} / \mathrm{cm}^{2}\right)\end{array}$ & $\begin{array}{c}\text { Concreto } \\
\text { clase 1 } \\
2.0 \times(\mathrm{fc}) / 2\end{array}$ & $\begin{array}{c}\text { Concreto } \\
\text { clase 2 } \\
1.4 \times(\mathrm{fc}) / 2\end{array}$ \\
\hline CN200 & 26.11 & 27.44 & 19.21 \\
CR200 & 29.26 & 27.24 & 19.07 \\
CN300 & 42.22 & 35.03 & 24.52 \\
CR300 & 39.89 & 34.68 & 24.28 \\
CN400 & 52.11 & 39.27 & 27.49 \\
CR400 & 41.58 & 37.97 & 26.58 \\
\hline
\end{tabular}

Como se observa en la tabla 12, nuevamente los concretos reciclados presentaron valores menores que los que alcanzaron los concretos naturales, a excepción del CR200 que fue mayor que su homólogo natural. Sin embargo, los valores que alcanzaron todas las mezclas de concreto estudiadas tienen líneas de tendencia que caben dentro de la clasificación de concretos clase uno en ambos tipos de concretos como se aprecia en la figura 9.

\section{Módulo de elasticidad}

Para obtener el módulo de elasticidad de las mezclas de concreto, se utilizó la norma ASTM C 469. De acuerdo con el RCDF el módulo de elasticidad puede estimarse como 14,000 veces la raíz cuadrada de fc para concretos clase uno y como 8,000 veces la raíz cuadrada de fc para concretos clase dos.

En la tabla 13 se presentan los valores alcanzados de módulo de elasticidad para los concretos estudiados.

En la misma, se observa que los concretos reciclados como en todas las propiedades mecánicas estudiadas, presentaron valores por debajo de los alcanzados por los concretos naturales.

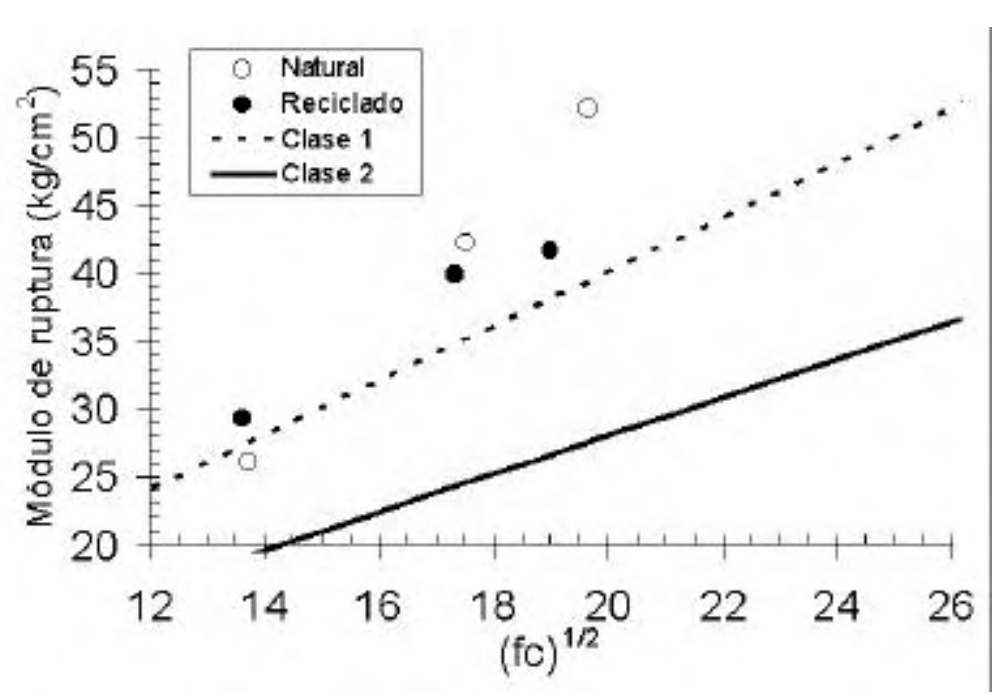

Figura 9. Resistencias a la flexión de las mezclas de concreto 
I.E. Martínez-Soto y C.J. Mendoza-Escobedo

Tabla 13. M ódulos de elasticidad de los concretos

\begin{tabular}{lccc}
\hline Mezcla & $\begin{array}{c}\text { Módulo de } \\
\text { elasticidad } \\
(\mathrm{E}) \times 103 \\
\left(\mathrm{~kg} / \mathrm{cm}^{2}\right)\end{array}$ & $\begin{array}{c}\text { Concreto } \\
\text { clase 1 } \\
14,000 \times(\mathrm{fc}){ }^{2 / 2} \\
\mathrm{E} \times 10^{3}\end{array}$ & $\begin{array}{c}\text { Concreto } \\
\text { clase 2 } \\
8,000 \times(\mathrm{fc}) / 2 \\
\mathrm{E} \times 10^{3}\end{array}$ \\
\hline CN200 & 227 & 192 & 110 \\
CR200 & 170 & 191 & 109 \\
CN300 & 263 & 245 & 140 \\
CR300 & 214 & 243 & 139 \\
CN400 & 264 & 275 & 157 \\
CR400 & 188 & 266 & 152 \\
\hline
\end{tabular}

Como se observa en la figura 10 , los valores alcanzados por los concretos reciclados tuvieron módulos de elasticidad superiores a los correspondientes a concretos de clase dos (RCDF), mientras que los concretos naturales se clasificaron dentro de los concretos clase 1.

Las deformaciones unitarias máximas que alcanzaron los concretos reciclados fueron del mismo orden que los naturales y se encontraron en el intervalo de 0.0029 a 0.0036 , como se muestra en la figura 11. Los trabajos disponibles en la literatura técnica hasta el momento, no presentan resultados comparables a los obtenidos en esta investigación, ya que las condiciones de los ensayes de otros autores (e.g., Katz, 2003) no son las mismas que las de este trabajo.

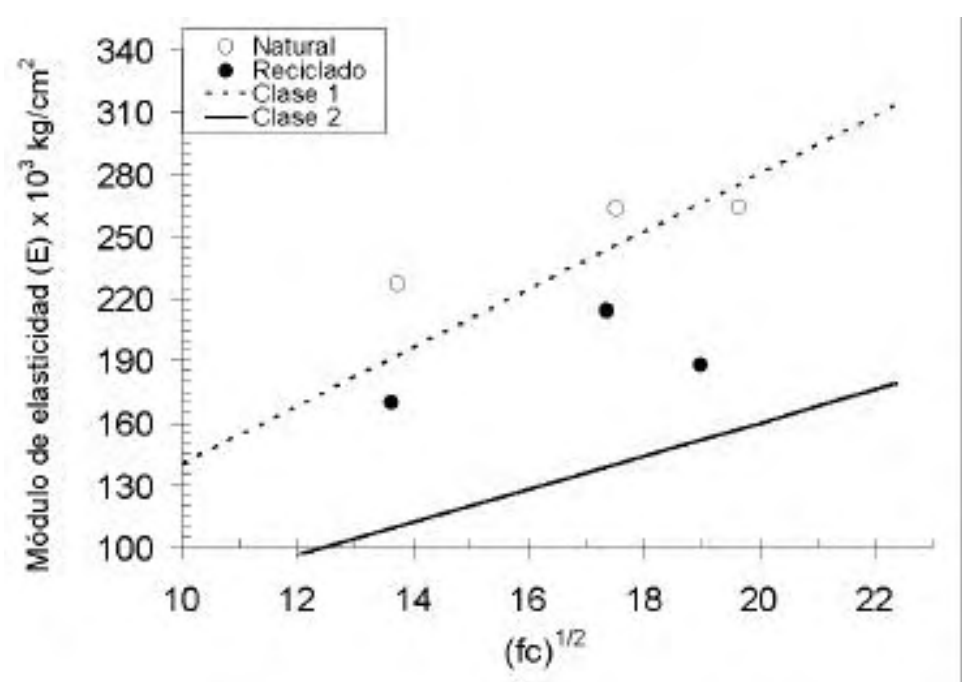

Figura 10. M ódulos de elasticidad de las mezclas de concreto

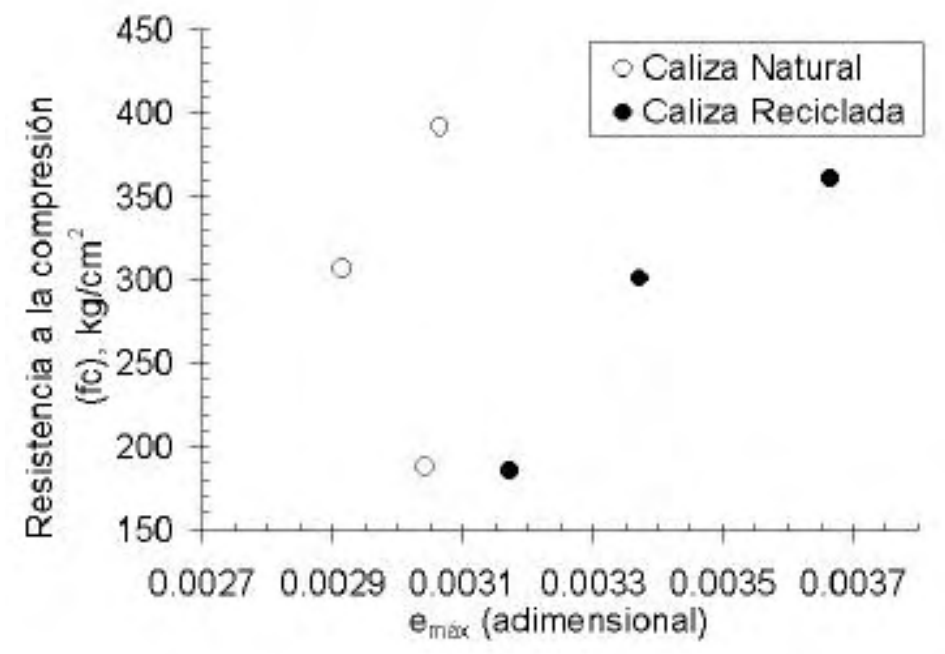

Figura 11. D eformaciones máximas unitarias de las mezclas de concreto 


\section{Conclusiones}

El reciclaje de concreto para fabricar agregado grueso y sustituir al natural es una práctica que debe empezar a realizarse a la brevedad posible, ya que la disponibilidad de bancos de materiales pétreos es cada día más escasa.

Este trabajo de investigación reveló que el agregado reciclado con granulometría adecuada produce mezclas de buena calidad y con un comportamiento mecánico similar al de los concretos naturales.

Los concretos reciclados pueden ser utilizados como concretos clase dos, lo que lo convierte en un concreto con una cantidad de aplicaciones nada despreciables.

En las resistencias a la tensión y flexión, se encontró que para consumos de cemento de 300 $\mathrm{kg} / \mathrm{m}^{3}$ y mayores, la relación $\mathrm{ft} / \mathrm{fc}{ }^{1 / 2}$ y MR/fc ${ }^{1 / 2}$ eran menores para los concretos reciclados, lo que se puede deber a que a bajas relaciones agua-cemento, domina el comportamiento del agregado grueso y a altas relaciones agua-cemento domina el de la pasta. Lo que conlleva a pensar que el agregado reciclado tiene su mejor aplicación en consumos de cemento bajos hasta $300 \mathrm{~kg} / \mathrm{m}^{3}$, debido a que para consumos mayores pueden resultar mezclas antieconómicas.

Por último, esta investigación constituye el punto de partida de investigaciones complementarias.

\section{Agradecimientos}

Este trabajo se realizó gracias a las facilidades prestadas por el IIUNAM para la realización de los experimentos en su laboratorio de estructuras y materiales.

Asimismo, se agradece el apoyo moral y económico que el Instituto brindó para la realización de esta investigación.

\section{Referencias}

Abdol R.C., Shiou-San K., Jamshid M.A., James

P.D. (2001). Test of Recycled Concrete
Aggre gate in Accel er ated Test Track.Journal ofTransportation Engineering, Vol. 127, No. 6, November/December, pp. 486-492.

$\mathrm{ACl} 211.1$ - 89. Stan dard Prac tice for Selecting Proportions Normal, Heavyweight, and Mass Concrete.

ASTM C 29/ C 29M - 90. Stan dard Test Method for Unit Weight and Voids in Aggre gate.

ASTM C 33 - 90. Standard Specification for Concrete Aggre gates.

ASTM C 39. Standard Test Method for Compressive Strength of Cylindrical Concretespecimens.

ASTM C 78 - 84. Standard Test Method for Flex ural Strength of Concrete (Using Simple Beam with Third-Point Loading).

ASTM C 94 - 90. Standard Specification for Ready-Mixed Concrete.

ASTM C 127 - 88. Standard Test Method for Specific Gravity and Absorption of Coarse Aggregate.

ASTM C 128 - 88. Standard Test Method for Specific Gravity and Absorption of Fine Aggregate.

ASTM C 136 - 84a. Stan dard Method for Sieve Anal ysis of Fine and Coarse Aggre gates.

ASTM C 138 - 81. Standard Test Method for Unit Weight, Yield, and Air Content (Gravimetric) of Concrete.

ASTM C 143 - 90a. Standard Test Method for Slump of Hydraulic Cement Concrete.

ASTM C 192 - 90a. Stan dard Prac tice for Making and Curing Test Spec i mens in the Labo ra tory.

ASTM C 231 - 91. Standard Test Method for Air Content of Freshly Mixed Concrete by the Pres sure Method.

ASTM C 469 - 87a. Standard Test Method for Static Modulus of Elasticity and Poisson's Ratio of Concrete in Compres sion.

ASTM C 496 - 90. Standard Test Method for Splitting Tensile Strength of Cylindrical ConcreteSpecimens.

ASTM C 511 - 85. Standard Specification for Moist Cabinets, Moist Rooms, and Water Storage Tanks Used in the Testing of Hydraulic Cements and Concretes.

ASTM C 566 - 89. Standard Method for Total Mois ture Content of Aggre gate by Drying.

ASTM C 617. Standard Practice for Capping Cylindrical ConcreteSpecimens. 
Comisión Federal de Electricidad (CFE), (1994). Manual de tecnología del concreto, sección 1. Ed. Limusa, México.

Comisión Federal de Electricidad (CFE), (1994). Manual de tecnología del concreto, sección 3. Ed. Limusa, México.

Katz A. (2003). Properties of Concrete Made With Recycled Aggregate From Partially Hydrated Old Concrete. Cement and Concrete Research, Vol. 33, pp. 703-711.

Kosmatka S.H., Panarese W.C. (1992). Diseño y control de mezclas de concreto. Ed. IMCyC, M éxico.

Martínez-Soto I.E. (2005). Reciclaje de concreto premezclado para la fabricación de agregados, Tesis de Maestría en Ingeniería, Universidad Nacional Autónoma de México, Ciudad Universitaria, México.

NMX C 414 - 2004. Industria de la construcción -cementos hidráulicos- especificaciones y métodos de prueba.

Reglamento de Construcciones para el Distrito Federal (2003). Ed. México Sista.

Sagoe-Crentsil K.K., Brown T., Taylor A.H. (2001). Perfor mance of Concrete Made with Commercially Produced Coarse Recycled Concrete Aggregate. Cement And Concrete Research, Vol. 31, pp. 707-712.

\section{Bibliografía sugerida}

Bjorn-Jakobse J., Elle M., Lauritzen E.K. (1988). On Site Use of Regen er ated Demo lition Debris. In: Kasai Y. (Ed.), Demolition and Reuse of Concrete and Masonry, Reuse of Demolition Waste, Vol. 2, E\&FN SPON, London, pp. 537-546.

Gómez-Soberón J.M.V. (2002). Porosity of Recycled Concrete with Substitution of Recycled Concrete Aggregate, an Experimental Study. Cement and Concrete Research, 32, pp. 1301-1311.

Hendriks C.F. (1994). Certification System for Aggregates Produced from Building Waste and Demolished Buildings. In: Goumans J.J.J.M., Van Der Sloot H.A., Aalbers T.G. (Eds.), Environmental Aspects of Construction with Waste Materials, Elsevier, Ams- terdam, The Netherlands, pp. 821-843.
How-Ji C., Tsong Y., Kuan-Hung C. (2003). Use of Building Rubbles as Recycled Aggregates. Cement and Concrete Research 33, pp. 125-132.

Kasai J., Kasai Y. (1993). Guidelines and the Present State of the Use of Demolished Concrete in Japan. In: Lauritzen E.K. (Ed.), Demolition and Reuse of Concrete and Masonry, Guidelines for Demolition and Reuse of Concrete and Masonry, E\&FN SPON, Denmark, pp. 93-104.

Luc T., Peter De P., Jan D. (1999). Concrete with Recycled Materials as Coarse Aggregates: Shrinkage and Creep Behavior, Materials and Construction: Exploring the Connec tion. Proceed ings of the Fifth ASCE Materials Engineering Congress held in Cincinnati, Ohio, May 10-12, pp. 720-727.

Nataatmadja A. and Tan Y.L. (2001). Resilient Response of Recycled Concrete Road Aggregates. Journal of Transportation Engineering, Vol. 127, No. 5, September/October, pp. 450-453.

Nixon P.J. (1978). Recycled Concrete as an Aggregate for Concrete - A Review, RILEM TC-37-DRC. M aterials and Structures (RILEM), 65, pp. 371-378.

Rashwan, M., and Aourizk, S. (1997). Factors Affecting Prop erties Of Concrete Made With Reclaimed $\mathrm{M}$ ate rial in Ready Mix Plants. $\mathrm{ACl}$ Concrete International, Vol. 10, No. 7, pp. 56-60.

Rashwan M. and Aourizk S. (1997). The Properties Of Recycled Concrete. ACI Concrete International, Vol. 19, No. 7, pp. 56-60.

RILEM 121-DRG (1994). Specification for Concrete With Recycled Aggre gates, Materials and Struc tures 27 (173), pp. 557-559.

Shiou-San K., Abdol R.C., and Jamshid M.A. (1999). Testing and Evaluation of Recycled Concrete Aggregate (ACR) as Mixture and Base $M$ ate rial, Mate rials and Construc tion: Exploring the Connection. Proceedings of the Fifth ASCE Materials Engineering Congress held in Cincinnati, Ohio, May 10-12, pp. 704-711.

Stamatia A., Frondistou-Yannas and Taichi I. (1977). Economic Feasibility of Concrete Recycling. Journal of the Structural Division, Vol. 103, No.4, April, pp. 885-899. 
Comportamiento mecánico de concreto fabricado con agregados reciclados

Vyncke J., Rousseau E. (1993). Recycling of Construction and Waste in Belgium: Actual Situa tion and Future Evolu tion. In: Lau- ritzen E.K. (Ed.),
Demolition and Reuse of Concrete and Masonry, Guidelines for Demolition and Reuse of Concrete and Masonry, E\&FN SPON, Denmark, pp. 57-69.

\section{Semblanza de los autores}

Iris Esmeralda Martínez-Soto. Obtuvo el título de ingeniera civil en la Escuela de Ingeniería y Arquitectura de la Universidad Autónoma de Sinaloa en el año de 2001. Cursó sus estudios de maestría en ingeniería (construcción) en la Universidad Nacional Autónoma de México, obteniendo el grado en el año 2005. A lo largo de tres años de carrera profesional como ingeniero civil, ha participado en diversos proyectos de desarrollo de infraestructura, tanto en el sector privado como en el público, en el área de construcción e ingeniería de costos. En la investigación, colaboró en el Instituto de Ingeniería de octubre de 2003 a agosto de 2005, como becaria, en proyectos de investigación dentro del área de concreto, relacionados con el distribuidor vial del Distrito Federal, la aplicación del concreto reciclado en edificacione s y sobre el aspecto térmico del uso del mate rial para el ahorro de energía en edificios de concreto. También ha dictado conferencias en el ACI México sobre el desarrollo de sus investigaciones. Asimismo, ha sido coautora en publicaciones del área de matemáticas aplicadas a la construcción y autora en los boletines informativos del ACI México. Actualmente se desempeña como coordinadora de precios unitarios en el Aeropuerto Internacional de la Ciudad de México.

Carlos Javier M endoza-Escobedo. Es ingeniero civil por la Universidad Autónoma de Yucatán y maestro en ingeniería por la Universidad Nacional Autóno ma de México. Es investigador del Instituto de Ingeniería desde 1971. Es secretario del Comité Asesor en Seguridad Estructural del Distrito Federal, pres i dente de la Comisión de Especialidad de Ingeniería Civil de la Academia de Ingeniería, miembro de la Comisión evaluadora del PRIDE de la ENEP Acatlán, UNAM y miembro del Consejo Técnico del Organismo Nacional de Normalización y Certificación de la Construcción. Sus campos de interés son la tecnología y las estructuras de concreto, y sobre estos temas, ha publicado 146 trabajos técnicos, ha participado en la elaboración de varias Normas Mexicanas y en la preparación de las nuevas Normas Técnicas Complementarias del Reglamento de Construcciones del Gobierno del Distrito Federal. 